\title{
Variabilidade de indicadores fisiológicos de resistência à salinidade entre genótipos de cajueiro-anão e gigante
}

\author{
Luiz Ferreira Aguiar Ponte(1), Osnan Soares Ferreira(2), Francisco Abel Lemos Alves ${ }^{(3)}$, \\ Sérgio Luiz Ferreira-Silva( ${ }^{(2)}$, Vanda Lúcia Arcanjo Pereira ${ }^{(3)}$ e Joaquim Albenísio Gomes da Silveira( ${ }^{(2)}$ \\ (1)Universidade Estadual Vale do Acaraú, Centro de Ciências Agrárias e Biológicas, Avenida da Universidade, no 850, Campus da Betânia, \\ CEP 62040-370 Sobral, CE. E-mail: luizponte200@yahoo.com.br (2)Universidade Federal do Ceará, Departamento de Bioquímica e Biologia \\ Molecular, Caixa Postal 6020, CEP 60451-970 Fortaleza, CE. E-mail: luizponte@hotmail.com, agrosergol@yahoo.com.br, silveira@ufc.br \\ (3)Instituto Agronômico de Pernambuco, Estação Experimental de Arcoverde, BR 232, Km 253, CEP $56500-000$ Arcoverde, PE. \\ E-mail: abel.alves@ipa.br, vanda.arcanjo@ipa.br
}

\begin{abstract}
Resumo - $\mathrm{O}$ objetivo deste trabalho foi avaliar a resistência à salinidade de genótipos de cajueiro dos grupos anão e gigante, em mudas em fase de enxertia, com o uso de indicadores fisiológicos. A germinação e o crescimento inicial de plântulas de dez genótipos de porta-enxertos foram avaliados sob duas condições de salinidade: irrigação do substrato com água destilada ou com solução de $\mathrm{NaCl} 50 \mathrm{mmol} \mathrm{L}^{-1}$, até o estádio de oito folhas maduras (28 dias após o plantio). As mudas foram irrigadas com solução nutritiva de Hoagland \& Arnon por mais dez dias. Os indicadores fisiológicos de resistência - concentrações de $\mathrm{Na}^{+}$e $\mathrm{K}^{+}$e relação $\mathrm{K}^{+} / \mathrm{Na}^{+}$, em folhas e raízes - não diferiram entre os genótipos, na ausência ou na presença de salinidade. Os indicadores e as concentrações de prolina não se correlacionaram com a massa acumulada em raízes e folhas. Sob salinidade, os genótipos do grupo anão apresentaram maior massa radicular em comparação aos do grupo gigante, que tiveram maior alocação de massa em folhas. Houve baixa variabilidade genética dentro de cada grupo. Quanto ao crescimento de raízes e folhas, houve variabilidade entre os grupos, nas duas condições de salinidade.
\end{abstract}

Termos para indexação: Anacardium occidentale, estresse salino, resistência à salinidade, variabilidade genética.

\section{Variability of physiological indicators of resistance to salinity between genotypes of dwarf and giant cashew}

\begin{abstract}
The objective of this work was to evaluate the resistance to salinity of cashew genotypes from dwarf and giant groups, with seedlings in pregrafting phase, using physiological indicators. Germination and seedling initial growth of ten rootstock genotypes were evaluated under two salinity conditions: substrate irrigated with distilled water or with 50 -mmol L-1 $\mathrm{NaCl}$ solution, until the eight mature leaves stage (28 days after sowing). The seedlings were irrigated with Hoagland \& Arnon nutrient solution, for ten more days. Physiological indicators of resistance $-\mathrm{Na}^{+}$and $\mathrm{K}^{+}$contents and $\mathrm{K}^{+} / \mathrm{Na}^{+}$relation, in leaves and roots - did not differ between genotypes, in the absence or presence of salinity. The indicators and prolin contents were not correlated to the accumulated mass in roots and leaves. Under salinity conditions, the genotypes from the dwarf group showed higher root mass in comparison to those from the giant group, which had greater mass allocation in leaves. There was low genetic variability within each group. Regarding root and leaf growth, there was variability between groups, in both salinity conditions.
\end{abstract}

Index terms: Anacardium occidentale, salt stress, salt resistance, genetic variability.

\section{Introdução}

A salinidade dos solos constitui um dos principais problemas ambientais que restringe a produtividade agrícola no mundo, e ocorre principalmente em regiões áridas e semiáridas (Munns, 2002). Atualmente, estima-se que dos 1.500 milhões de hectares de terras cultivadas pela agricultura de sequeiro, cerca de 32 milhões de hectares (2\%) são afetados pela salinidade em diferentes intensidades (Munns \&
Tester, 2008). Esse problema é ainda mais grave com relação às áreas agrícolas irrigadas, que apresentam problemas de salinidade em pelo menos $33 \%$ do seu total (Munns, 2005). A cultura do cajueiro irrigado tem se expandido no Nordeste brasileiro, em função do aumento expressivo da produtividade da cultura pelo uso dessa técnica (Oliveira et al., 2002).

Entre os mecanismos mais conhecidos relacionados à resistência à salinidade, está a capacidade de exclusão de $\mathrm{Na}^{+} \mathrm{e} \mathrm{Cl}$, que é fundamental para a caracterização da 
resistência ao estresse salino em espécies ou genótipos de interesse (Wahome et al., 2001; Estañ et al., 2005; Paranychianakis \& Angelakis, 2008). A salinidade é responsável pela alteração do estado nutricional das plantas, evidenciado por mudanças nas relações $\mathrm{K}^{+} / \mathrm{Na}^{+}, \mathrm{Ca}^{++} / \mathrm{Na}^{+}$e $\mathrm{NO}_{3}^{-} / \mathrm{Cl}^{-}$nos tecidos (Apse \& Blumwald, 2007). Uma maior relação $\mathrm{K}^{+} / \mathrm{Na}^{+}$no tecido de plantas, sob estresse salino, é considerada como um importante marcador de resistência à salinidade (Dasgan et al., 2002; Estañ et al., 2005). A acumulação de $\mathrm{K}^{+}$e de outros solutos compatíveis na célula vegetal, tais como prolina, glicina betaína e açúcares (Silva et al., 2009), é considerada um importante mecanismo bioquímico e fisiológico envolvido com a resistência à salinidade (Ashraf \& Harris, 2004; Ashraf \& Foolad, 2007).

O cajueiro (Anacardium occidentale L.) é uma espécie amplamente cultivada em áreas sujeitas à salinização no Semiárido do Nordeste brasileiro, onde sua cadeia produtiva representa uma importante fonte de emprego e renda (Barros et al., 2000). Para o cultivo nessas áreas, emprega-se genótipos de cajueiro-anão precoce e gigante. O tipo anão precoce é representado por diversos clones já bem caracterizados para utilização como enxertos e porta-enxertos em plantios comerciais, em diversas áreas da região (Barros et al., 2000, 2002; Paiva et al., 2002). A identificação de enxertos e porta-enxertos do tipo gigante é ainda bastante reduzida (Paiva et al., 2008), principalmente no que se refere à disponibilidade desses materiais para o cultivo em áreas salinizadas.

Os diferentes genótipos de cajueiro-anão precoce atualmente disponíveis para o plantio comercial apresentam baixa variabilidade genética quanto a diferentes caracteres de importância agronômica(Barros et al., 2000, 2002; Paiva et al., 2005). No entanto, estudos recentes têm demonstrado a ocorrência de variabilidade genética nesse tipo de cajueiro, em relação à resistência à salinidade (Ferreira-Silva et al., 2010). Essa diversidade está associada à capacidade de exclusão de íons salinos $\left(\mathrm{Na}^{+} \mathrm{e} \mathrm{Cl}^{-}\right)$e às mudanças favoráveis na concentração de solutos orgânicos compatíveis, tanto em plântulas (Ferreira-Silva et al., 2008) quanto em mudas enxertadas (Ferreira-Silva et al., 2009, 2010).

Os resultados são animadores e indicam a possibilidade da identificação de genótipos do grupo de cajueiro-anão, com potencial para serem utilizados em áreas salinizadas. Não se tem conhecimento de estudos voltados à identificação de genótipos com caracteres favoráveis de resistência à salinidade no grupo gigante (Paiva et al., 2008). Estudos que visem identificar variabilidade genética, assim como marcadores fisiológicos de resistência nesses materiais, para a seleção de genótipos tolerantes à salinidade, podem contribuir para a sustentabilidade da cultura em áreas propensas à salinização, como as do Semiárido brasileiro.

O objetivo deste trabalho foi avaliar a resistência de genótipos de cajueiro dos grupos anão e gigante à salinidade, em mudas em fase de enxertia, com uso de indicadores fisiológicos.

\section{Material e Métodos}

O trabalho foi realizado em casa de vegetação do Departamento de Bioquímica e Biologia Molecular da Universidade Federal do Ceará, Fortaleza, CE ( $3^{\circ} 45^{\prime} 47^{\prime \prime} \mathrm{S}, 38^{\circ} 31^{\prime} 23^{\prime \prime} \mathrm{W}$, altitude ao nível do mar). Durante o experimento, as plântulas estiveram expostas a valores médios de temperaturas de $29 \pm 4^{\circ} \mathrm{C}$, dia, e $25 \pm 2^{\circ} \mathrm{C}$, noite, umidade relativa do ar de $60 \pm 10 \%$, radiação fotossinteticamente ativa de $1.200 \pm 200 \mu \mathrm{mol} \mathrm{m}^{-2} \mathrm{~s}^{-1}$ e fotoperíodo de 12 horas de luz, característicos do Semiárido nordestino.

As plântulas de porta-enxerto foram obtidas a partir de castanhas dos clones comerciais de cajueiro-anão precoce (CCP 06, СCP 09, СCP 76, СCP 1001, EMBRAPA 50 e EMBRAPA 51) e de progênies de cajueiro-gigante (CP 49, CP 95, CP 96 e Comum), cedidas pela Embrapa Agroindústria Tropical. As castanhas foram desinfetadas em hipoclorito de sódio comercial a $5 \%(\mathrm{v} / \mathrm{v})$ por $10 \mathrm{~min}$ e, em seguida, lavadas com água destilada e colocadas para germinar em tubetes de plástico, de uso comercial, com $19 \mathrm{~cm}$ de altura, $6,3 \mathrm{~cm}$ de diâmetro e volume de $288 \mathrm{~cm}^{3}$, contendo vermiculita como substrato, em condições de casa de vegetação. Os tubetes foram suspensos em bandejas apropriadas, com capacidade para 54 tubetes cada uma, instaladas sobre estruturas metálicas a $1 \mathrm{~m}$ de altura.

Durante a germinação e o crescimento inicial das plântulas, a umidade do substrato foi mantida por meio de irrigações, a cada dois dias, com água destilada (tratamento controle) ou solução de $\mathrm{NaCl} 50 \mathrm{mmol} \mathrm{L}^{-1}$ (tratamento salino), até o estádio de oito folhas maduras (28 dias após o plantio). As mudas foram irrigadas 
com solução nutritiva de Hoagland \& Arnon (1950), em ambos os tratamentos, durante dez dias. Ao final do experimento - em mudas com 38 dias após o plantio, em fase de enxertia -, foi determinada a massa de matéria fresca (MF) de raízes, caules e folhas. As raízes e as folhas foram congeladas em $\mathrm{N}_{2}$ líquido e armazenadas a $-80^{\circ} \mathrm{C}$ e, em seguida, liofilizadas. A farinha do tecido foi obtida pela maceração em almofariz e utilizada na determinação dos solutos. As concentrações de $\mathrm{Na}^{+}$ $\mathrm{e}^{+}$foram determinadas por fotometria de chama e expressas em mmol kg-1 de massa de matéria seca (MS), de acordo com Viégas et al. (2001). A concentração de prolina livre foi mensurada conforme o método de Bates (1973), com curva padrão de prolina, e os valores foram expressos em mmol kg-1 de MS.

$\mathrm{O}$ experimento foi realizado em delineamento inteiramente casualizado, com tratamentos dispostos em arranjo fatorial $10 \times 2$, com dez porta-enxertos - seis do tipo anão e quatro do gigante -, duas concentrações de $\mathrm{NaCl}\left(0\right.$ e $\left.50 \mathrm{mmol} \mathrm{L}^{-1}\right)$, e três repetições por tratamento. A parcela experimental foi representada por quatro tubetes com uma planta em cada um. Os dados foram submetidos à análise de variância e, quando o teste $\mathrm{F}$ foi significativo, as médias foram comparadas pelo teste de Tukey, a 5\% de probabilidade.

\section{Resultados e Discussão}

Não foram observadas diferenças na concentração de $\mathrm{Na}^{+}$, nas raízes ou nas folhas, entre os genótipos, na ausência (controle) ou na presença de $\mathrm{NaCl}$ (Tabela 1). A concentração de $\mathrm{Na}^{+}$foi cerca de dez vezes maior nas plântulas salinizadas, em comparação ao controle. No tratamento controle, a concentração de $\mathrm{K}^{+}$, nas raízes e nas folhas, não diferiu entre os genótipos. No tratamento com $\mathrm{NaCl}$, a concentração de $\mathrm{K}^{+}$foliar não foi afetada; porém, observou-se intensa redução na concentração desse nutriente nas raízes, especialmente no genótipo EMBRAPA 51.

Tabela 1. Concentrações de $\mathrm{Na}^{+}$e $\mathrm{K}^{+}$(em mmol kg-1 de massa de matéria seca) e relação $\mathrm{K}^{+} / \mathrm{Na}^{+}$em tecidos de raízes e de folhas de seis clones de cajueiro-anão precoce (CCP 06, CCP 09, CCP 76, CCP 1001, EMBRAPA 50 e EMBRAPA 51) e de quatro progênies de cajueiro-gigante (CP 49, CP 95, CP 96 e Comum), 38 dias após o plantio, na ausência (controle) ou na presença de $\mathrm{NaCl}\left(50 \mathrm{mmol} \mathrm{L}^{-1}\right)^{(1)}$.

\begin{tabular}{|c|c|c|c|c|c|c|}
\hline \multirow[t]{2}{*}{ Genótipo } & \multicolumn{2}{|c|}{$\mathrm{Na}^{+}$} & \multicolumn{2}{|c|}{$\mathrm{K}^{+}$} & \multicolumn{2}{|c|}{$\mathrm{K}^{+} / \mathrm{Na}^{+}$} \\
\hline & Controle & $\mathrm{NaCl}$ & Controle & $\mathrm{NaCl}$ & Controle & $\mathrm{NaCl}$ \\
\hline & \multicolumn{6}{|c|}{ Raiz } \\
\hline ССР 06 & $170 \mathrm{aB}$ & $1.800 \mathrm{aA}$ & $451 \mathrm{aA}$ & $90 \mathrm{abB}$ & $2,66 \mathrm{abA}$ & $0,05 \mathrm{aB}$ \\
\hline ССР 09 & $143 \mathrm{aB}$ & $2.035 \mathrm{aA}$ & $474 \mathrm{aA}$ & $105 \mathrm{aB}$ & $3,31 \mathrm{aA}$ & $0,05 \mathrm{aB}$ \\
\hline CCP 76 & $183 \mathrm{aB}$ & $1.839 \mathrm{aA}$ & $441 \mathrm{aA}$ & $103 \mathrm{aB}$ & $2,42 \mathrm{bA}$ & $0,06 \mathrm{aB}$ \\
\hline CCP 1001 & $196 \mathrm{aB}$ & $1.957 \mathrm{aA}$ & $459 \mathrm{aA}$ & $126 \mathrm{aB}$ & $2,35 \mathrm{bA}$ & $0,06 \mathrm{aB}$ \\
\hline EMBRAPA 50 & $183 \mathrm{aB}$ & $1.865 \mathrm{aA}$ & $431 \mathrm{aA}$ & $87 \mathrm{abB}$ & $2,36 \mathrm{bA}$ & $0,05 \mathrm{aB}$ \\
\hline EMBRAPA 51 & $222 \mathrm{aB}$ & $2.048 \mathrm{aA}$ & $423 \mathrm{aA}$ & $59 b B$ & $1,91 \mathrm{bA}$ & $0,03 \mathrm{bB}$ \\
\hline CP 49 & $196 \mathrm{aB}$ & $1.957 \mathrm{aA}$ & $444 \mathrm{aA}$ & $85 \mathrm{abB}$ & $2,27 \mathrm{bA}$ & $0,04 \mathrm{aB}$ \\
\hline CP 95 & $209 a B$ & $2.061 \mathrm{aA}$ & $449 \mathrm{aA}$ & $82 \mathrm{abB}$ & $2,15 \mathrm{bA}$ & $0,04 \mathrm{aB}$ \\
\hline CP 96 & $196 \mathrm{aB}$ & $2.035 \mathrm{aA}$ & $423 \mathrm{aA}$ & $118 \mathrm{aB}$ & $2,16 \mathrm{bA}$ & $0,06 \mathrm{aB}$ \\
\hline Comum & $183 \mathrm{aB}$ & $2.061 \mathrm{aA}$ & $449 \mathrm{aA}$ & $108 \mathrm{aB}$ & $2,46 \mathrm{bA}$ & $0,05 \mathrm{aB}$ \\
\hline \multirow[t]{2}{*}{ DMS } & 52,37 & 275,58 & 47,21 & 23,27 & 0,39 & 0,013 \\
\hline & \multicolumn{6}{|c|}{ Folha } \\
\hline ССР 06 & $143 \mathrm{aB}$ & $1.591 \mathrm{aA}$ & $467 \mathrm{aA}$ & $462 \mathrm{aA}$ & $3,25 \mathrm{bA}$ & $0,29 \mathrm{aB}$ \\
\hline ССР 09 & $117 \mathrm{aB}$ & $1.643 \mathrm{aA}$ & $477 \mathrm{aA}$ & $503 \mathrm{aA}$ & $4,06 \mathrm{aA}$ & $0,31 \mathrm{aB}$ \\
\hline ССР 76 & $130 \mathrm{aB}$ & $1.735 \mathrm{aA}$ & $467 \mathrm{aA}$ & $492 \mathrm{aA}$ & $3,58 \mathrm{abA}$ & $0,28 \mathrm{aB}$ \\
\hline CCP 1001 & $143 \mathrm{aB}$ & $1.657 \mathrm{aA}$ & $469 \mathrm{aA}$ & $495 \mathrm{aA}$ & $3,27 \mathrm{bA}$ & $0,30 \mathrm{aB}$ \\
\hline EMBRAPA 50 & $157 \mathrm{aB}$ & $1.735 \mathrm{aA}$ & $464 \mathrm{aA}$ & $490 \mathrm{aA}$ & $2,97 \mathrm{cA}$ & $0,28 \mathrm{aB}$ \\
\hline EMBRAPA 51 & $117 \mathrm{aB}$ & $1.865 \mathrm{aA}$ & $479 \mathrm{aA}$ & $487 \mathrm{aA}$ & $4,08 \mathrm{aA}$ & $0,26 \mathrm{aB}$ \\
\hline CP 49 & $143 \mathrm{aB}$ & $1.930 \mathrm{aA}$ & $441 \mathrm{aA}$ & $456 \mathrm{aA}$ & $3,07 \mathrm{bA}$ & $0,24 \mathrm{aB}$ \\
\hline CP 95 & $157 \mathrm{aB}$ & $2.009 \mathrm{aA}$ & $438 \mathrm{aA}$ & $451 \mathrm{aA}$ & $2,80 \mathrm{cA}$ & $0,22 \mathrm{aB}$ \\
\hline CP 96 & $157 \mathrm{aB}$ & $1.943 \mathrm{aA}$ & $444 \mathrm{aA}$ & $459 \mathrm{aA}$ & $2,83 \mathrm{cA}$ & $0,24 \mathrm{aB}$ \\
\hline Comum & $157 \mathrm{aB}$ & $1.970 \mathrm{aA}$ & $449 \mathrm{aA}$ & $456 \mathrm{aA}$ & $2,87 \mathrm{cA}$ & $0,23 \mathrm{aB}$ \\
\hline DMS & 31,17 & 423,68 & 45,78 & 57,69 & 0,24 & 0,06 \\
\hline
\end{tabular}

${ }^{(1)}$ Médias seguidas de letras iguais, maiúsculas nas colunas e minúsculas nas linhas, não diferem pelo teste de Tukey, a 5\% de probabilidade. 
$\mathrm{Na}$ ausência de salinidade, a relação $\mathrm{K}^{+} / \mathrm{Na}^{+}$nas raízes foi maior nos genótipos CCP 09 e CCP 06 (Tabela 1). Essa relação foi intensamente reduzida pela salinidade, cerca de 50 vezes menor, em todos os genótipos; entretanto, a redução foi significantemente maior nas raízes do genótipo EMBRAPA 51. No tecido foliar, a relação $\mathrm{K}^{+} / \mathrm{Na}^{+}$foi maior nos genótipos CCP 09, CCP 76 e EMBRAPA 51 e foi reduzida pela salinidade, em cerca de dez vezes, em todos os genótipos avaliados. Sob condições salinas, o padrão de acumulação de $\mathrm{Na}^{+} \mathrm{e} \mathrm{Cl}^{-}$na célula e nos tecidos da planta é determinante para a resistência ou a sensibilidade ao estresse. A inclusão dos íons nos vacúolos (Zhu, 2003), associada a mecanismos de restrição da absorção ou da retenção desses íons pelas raízes, pode evitar seus efeitos tóxicos em tecidos fotossinteticamente ativos e contribuir para o aumento da tolerância das plantas ao excesso salino (Wahome et al., 2001; Estañ et al., 2005; Colla et al., 2010).

Os diferentes genótipos de cajueiro avaliados não apresentaram diferenças quanto à capacidade de retenção do $\mathrm{Na}^{+}$nas raízes, sob salinidade. $\mathrm{O}$ acúmulo preferencial de $\mathrm{Na}^{+}$em raízes de plantas de laranja (García-Sanchez et al., 2002) e rosas (Wahome et al., 2001), sob condições salinas, atua como mecanismo de retenção e restringe seus efeitos tóxicos na parte aérea, com atenuação da necrose de tecidos fotossintéticos e favorecimento das trocas gasosas e da eficiência de uso da água.

O tratamento salino afetou severamente o estado nutricional do potássio de todos os genótipos avaliados. A salinidade causa redução da concentração de $\mathrm{K}^{+}$em raízes de cajueiro e está associada a uma drástica redução de crescimento desse órgão (Viégas et al., 2001; Alves et al., 2008; Ferreira-Silva et al., 2008). Dos genótipos de cajueiro avaliados, o EMBRAPA 51 apresentou a maior redução na concentração de $\mathrm{K}^{+}$e na relação $\mathrm{K}^{+}$/ $\mathrm{Na}^{+}$nas raízes, sob salinidade (Tabela 1). A relação $\mathrm{K}^{+} / \mathrm{Na}^{+}$acima de 1 , na célula vegetal, é necessária para muitos processos metabólicos dependentes do $\mathrm{K}^{+}$, como a síntese proteica, abertura estomática e balanço de cargas no citosol (Apse et al., 2007). Em cajueiro, diversos estudos têm demonstrado que a salinidade induz distúrbios metabólicos relacionados à mobilização de reservas cotiledonares (Voigt et al., 2009), à homeostase iônica (Viégas et al., 2001) e ao metabolismo do nitrogênio (Silveira et al., 2003; Viégas et al., 2004; Ferreira-Silva et al., 2008).
Sob condições não salinas, a concentração de prolina em raízes foi similar entre os genótipos avaliados (Tabela 2); no entanto, apresentou intenso aumento nas raízes de todos os genótipos em resposta à salinidade, com exceção do CCP 09. Esse aumento foi maior $(p<0,05)$ nos genótipos do grupo de cajueiro-gigante, em comparação aos genótipos do grupo anão. Em condições salinas, a concentração de prolina foliar foi menor no genótipo CCP 09. A salinidade causou um intenso aumento da concentração de prolina nas folhas nos genótipos avaliados, mas esse aumento foi maior $(\mathrm{p}<0,05)$ nos genótipos CP 95 e CP 96 do grupo gigante, e CCP 09, CCP 76 e EMBRAPA 51 do grupo anão.

A prolina é um osmosoluto compatível que pode contribuir para o ajustamento osmótico, bem como atuar na proteção de estruturas celulares sob condições de estresses abióticos (Ashraf \& Harris, 2004). A acumulação de prolina pode ocorrer em diversas espécies, sob salinidade (Silva et al., 2009) e seca (Silva et al., 2010), e esse acúmulo têm sido considerado como um indicador de resistência à esses estresses (Ashraf \& Harris, 2004). Para algumas espécies, o aumento da concentração de prolina em resposta à salinidade é atribuído mais ao desbalanço metabólico induzido pelo estresse do que à proteção celular (Lutts et al., 1999). Em folhas de cajueiro submetidas ao estresse salino,

Tabela 2. Concentrações de prolina (em mmol kg-1 de massa de matéria seca), em tecidos de raízes e de folhas de seis clones de cajueiro-anão precoce (CCP 06, CCP 09, CCP 76, CCP 1001, EMBRAPA 50 e EMBRAPA 51) e de quatro progênies de cajueiro-gigante (CP 49, CP 95, CP 96 e Comum), 38 dias após o plantio, na ausência (controle) ou na presença de $\mathrm{NaCl}\left(50 \mathrm{mmol} \mathrm{L}^{-1}\right)^{(1)}$.

\begin{tabular}{lccccc}
\hline Genótipo & \multicolumn{2}{c}{ Raiz } & & \multicolumn{2}{c}{ Folha } \\
\cline { 2 - 3 } \cline { 5 - 6 } & Controle & NaCl & & Controle & NaCl \\
\hline CCP 06 & $1,42 \mathrm{aB}$ & $2,31 \mathrm{bcA}$ & & $1,45 \mathrm{abB}$ & $5,49 \mathrm{bA}$ \\
CCP 09 & $1,55 \mathrm{aA}$ & $1,77 \mathrm{cA}$ & & $0,97 \mathrm{bB}$ & $7,12 \mathrm{abA}$ \\
CCP 76 & $1,65 \mathrm{aB}$ & $2,76 \mathrm{bA}$ & & $1,69 \mathrm{aB}$ & $8,59 \mathrm{abA}$ \\
CCP 1001 & $1,55 \mathrm{aB}$ & $2,25 \mathrm{bcA}$ & & $1,68 \mathrm{aB}$ & $4,68 \mathrm{bA}$ \\
EMBRAPA 50 & $1,58 \mathrm{aB}$ & $2,30 \mathrm{bcA}$ & & $1,92 \mathrm{aB}$ & $5,92 \mathrm{bA}$ \\
EMBRAPA 51 & $1,64 \mathrm{aB}$ & $2,21 \mathrm{bcA}$ & & $1,65 \mathrm{aB}$ & $6,65 \mathrm{abA}$ \\
CP 49 & $1,76 \mathrm{aB}$ & $3,53 \mathrm{aA}$ & & $1,94 \mathrm{aB}$ & $4,94 \mathrm{bA}$ \\
CP 95 & $1,67 \mathrm{aB}$ & $3,95 \mathrm{aA}$ & & $1,17 \mathrm{abB}$ & $11,17 \mathrm{aA}$ \\
CP 96 & $1,76 \mathrm{aB}$ & $3,51 \mathrm{aA}$ & & $1,46 \mathrm{aB}$ & $6,46 \mathrm{abA}$ \\
Comum & $1,59 \mathrm{aB}$ & $3,41 \mathrm{aA}$ & & $1,41 \mathrm{aB}$ & $4,91 \mathrm{bA}$ \\
\hline DMS & 0,31 & 0,27 & & 0,11 & 0,87 \\
\hline
\end{tabular}

${ }^{(1)}$ Médias seguidas de letras iguais, maiúsculas nas colunas e minúsculas nas linhas, não diferem pelo teste de Tukey, a 5\% de probabilidade. 
foi demonstrado que o aumento da concentração de prolina está mais associado ao desbalanço do metabolismo do nitrogênio, como assimilação de nitrato e degradação de proteína, do que à proteção celular (Silveira et al., 2003). Resultados similares, em que o acúmulo de prolina não apresentou relação com a resistência ao sal, foram observados em plântulas dos genótipos de cajueiro-anão CCP 06 e CCP 09 (Ferreira-Silva et al., 2008) e CCP 1001 (Viégas et al., 2004), e em mudas enxertadas dos genótipos CCP 09, CCP 06, CAPI 4 e BRS 226 (Ferreira-Silva et al., 2009, 2010).

A MF de raízes, um importante indicador de crescimento, foi similar entre os genótipos, nas mudas cultivadas na ausência do estresse salino (Tabela 3). Nas mudas expostas ao sal, houve uma forte redução da MF de raízes, que foi maior $(p<0,05)$ nos genótipos do grupo de cajueiro-gigante. Nos genótipos de cajueiro-gigante avaliados, o sistema radicular foi mais afetado pela salinidade do que nos genótipos de cajueiro-anão.

A MF de caules não foi afetada pela salinidade e não diferiu entre os genótipos, cultivados tanto na ausência como na presença do estresse salino (Tabela 3 ). Os diferentes genótipos avaliados não apresentaram diferenças significativas de MF foliar na ausência da salinidade. $O$ estresse salino causou uma intensa redução da MF de folhas em todos os genótipos; essa redução foi maior $(p<0,05)$ nos genótipos do grupo de cajueiro-anão precoce do que nos genótipos do grupo de cajueiro-gigante. A relação entre a MF de folhas e raízes (relação folha/raiz) foi maior nos genótipos de cajueiro-gigante, na ausência e na presença do estresse salino.

O grupo gigante apresenta maior sensibilidade do sistema radicular e maior resistência da parte aérea ao estresse salino (Tabela 4). A diferença no crescimento desses órgãos não pode ser atribuída a diferenças na acumulação e partição de $\mathrm{Na}^{+}$e $\mathrm{K}^{+}$, como demonstrado pela similaridade das concentrações de $\mathrm{Na}^{+}, \mathrm{K}^{+}$e relação $\mathrm{K}^{+} / \mathrm{Na}^{+}$na ausência e presença do sal nos dois grupos. A maior concentração de prolina em raízes de genótipos do grupo gigante, associada à maior restrição de crescimento observada nesses genótipos, pode indicar que a sensibilidade ao estresse está condicionada ao maior distúrbio metabólico causado pelo sal em suas raízes, de acordo com o proposto por Silveira et al. (2003).

Segundo a análise da alocação de MF entre folhas e raízes dos genótipos dos grupos de cajueiro-anão e gigante, sob condições não salinas, no grupo anão, essa partição é aproximadamente igual para os dois órgãos (raiz com 3,51 g e folha com 3,54 g por planta), enquanto, no grupo gigante, ocorre predominância de alocação de massa na parte aérea (raiz com 3,52 g e folha com 4,50 g por planta), (Figura 1 e Tabela 4). Essa partição foi alterada em resposta à salinidade e ocorreu de forma diferenciada entre os genótipos dos dois grupos de cajueiro. Sob condições salinas, a alocação de massa nos genótipos do grupo anão ocorreu preferencialmente na raiz (raiz com 2,14 g e folha com 1,47 g por planta); já no grupo gigante, a alocação de

Tabela 3. Massa de matéria fresca (em g por planta) de raízes, caules, folhas, e relação entre massa de matéria fresca na folha e raiz, de seis clones de cajueiro-anão precoce (CCP 06, CCP 09, CCP 76, CCP 1001, EMBRAPA 50 e EMBRAPA 51) e de quatro progênies de cajueiro-gigante (CP 49, CP 95, CP 96 e Comum), 38 dias após o plantio, na ausência (controle) ou na presença de $\mathrm{NaCl}\left(50 \mathrm{mmol} \mathrm{L}^{-1}\right)^{(1)}$.

\begin{tabular}{|c|c|c|c|c|c|c|c|c|}
\hline \multirow[t]{2}{*}{ Genótipo } & \multicolumn{2}{|c|}{ Raiz } & \multicolumn{2}{|c|}{ Caule } & \multicolumn{2}{|c|}{ Folha } & \multicolumn{2}{|c|}{ Folha/Raiz } \\
\hline & Controle & $\mathrm{NaCl}$ & Controle & $\mathrm{NaCl}$ & Controle & $\mathrm{NaCl}$ & Controle & $\mathrm{NaCl}$ \\
\hline CCP 06 & $3,65 \mathrm{aA}$ & $2,25 \mathrm{aB}$ & $3,66 \mathrm{aA}$ & $3,06 \mathrm{aA}$ & $3,48 \mathrm{aA}$ & $1,48 \mathrm{bB}$ & $0,95 \mathrm{bA}$ & $0,66 \mathrm{bB}$ \\
\hline ССР 09 & $3,53 \mathrm{aA}$ & $2,21 \mathrm{aB}$ & $3,84 \mathrm{aA}$ & $3,20 \mathrm{aA}$ & $3,50 \mathrm{aA}$ & $1,50 \mathrm{bB}$ & $0,99 \mathrm{bA}$ & $0,68 \mathrm{bB}$ \\
\hline CCP 76 & $3,46 \mathrm{aA}$ & $2,01 \mathrm{aB}$ & $3,80 \mathrm{aA}$ & $3,17 \mathrm{aA}$ & $3,63 \mathrm{aA}$ & $1,63 \mathrm{bB}$ & $1,05 \mathrm{bA}$ & $0,81 \mathrm{bB}$ \\
\hline CCP 1001 & $3,52 \mathrm{aA}$ & $2,20 \mathrm{aB}$ & $3,75 \mathrm{aA}$ & $3,13 \mathrm{aA}$ & $3,73 \mathrm{aA}$ & $1,63 \mathrm{bB}$ & $1,06 \mathrm{bA}$ & $0,79 \mathrm{bB}$ \\
\hline EMBRAPA 50 & $3,46 \mathrm{aA}$ & $2,16 \mathrm{aB}$ & $2,80 \mathrm{aA}$ & $2,80 \mathrm{aA}$ & $3,53 \mathrm{aA}$ & $1,23 \mathrm{bB}$ & $1,02 \mathrm{bA}$ & $0,57 \mathrm{bB}$ \\
\hline EMBRAPA 51 & $3,42 \mathrm{aA}$ & $2,01 \mathrm{aB}$ & $3,76 \mathrm{aA}$ & $3,04 \mathrm{aA}$ & $3,65 \mathrm{aA}$ & $1,25 \mathrm{bB}$ & $1,07 \mathrm{bA}$ & $0,62 \mathrm{bB}$ \\
\hline CP 49 & $3,45 \mathrm{aA}$ & $1,25 \mathrm{bB}$ & $3,77 \mathrm{aA}$ & $3,98 \mathrm{aA}$ & $4,32 \mathrm{aA}$ & $2,52 \mathrm{aB}$ & $1,25 \mathrm{aB}$ & $1,86 \mathrm{aA}$ \\
\hline CP 95 & $3,53 \mathrm{aA}$ & $1,13 \mathrm{bB}$ & $3,70 \mathrm{aA}$ & $3,92 \mathrm{aA}$ & $4,61 \mathrm{aA}$ & $2,61 \mathrm{aB}$ & $1,31 \mathrm{aB}$ & $2,31 \mathrm{aA}$ \\
\hline СР 96 & $3,56 \mathrm{aA}$ & $1,16 \mathrm{bB}$ & $3,57 \mathrm{aA}$ & $3,56 \mathrm{aA}$ & $4,49 \mathrm{aA}$ & $2,49 \mathrm{aB}$ & $1,26 \mathrm{aB}$ & $2,15 \mathrm{aA}$ \\
\hline Comum & $3,54 \mathrm{aA}$ & $1,34 \mathrm{bB}$ & $3,67 \mathrm{aA}$ & $3,64 \mathrm{aA}$ & $4,56 \mathrm{aA}$ & $2,56 \mathrm{aB}$ & $1,29 \mathrm{aB}$ & $1,91 \mathrm{aA}$ \\
\hline DMS & 0,62 & 0,28 & 1,12 & 1,25 & 1,19 & 0,41 & 0,07 & 0,25 \\
\hline
\end{tabular}

${ }^{(1)}$ Médias seguidas de letras iguais, maiúsculas nas colunas e minúsculas nas linhas, não diferem pelo teste de Tukey, a $5 \%$ de probabilidade. 
massa foi intensificada na parte aérea (raiz com $1,22 \mathrm{~g}$ e folha com 2,50 g por planta). A massa de matéria fresca média de raízes em plantas do grupo cajueiroanão precoce, na ausência (controle) e na presença

Tabela 4. Análise de 12 variáveis relacionadas à resistência à salinidade entre grupos $^{(1)}$ de cajueiro-anão precoce (clones CCP 06, CCP 09, CCP 76, CCP 1001, EMBRAPA 50 e EMBRAPA 51) e cajueiro-gigante (progênies CP 49, CP 95, CP 96 e Comum), 38 dias após o plantio, na ausência (controle) ou na presença de $\mathrm{NaCl}$ $\left(50 \mathrm{mmol} \mathrm{L}^{-1}\right)^{(2)}$.

\begin{tabular}{|c|c|c|c|c|c|}
\hline \multirow[t]{2}{*}{ Variável } & \multicolumn{2}{|c|}{ Grupo cajueiro-anão } & \multicolumn{2}{|c|}{ Grupo cajueiro-gigante } & \multirow[t]{2}{*}{ DMS } \\
\hline & Controle & $\mathrm{NaCl}$ & Controle & $\mathrm{NaCl}$ & \\
\hline$\overline{\mathrm{MF}^{(3)} \text { de raiz }}$ & $3,51 \mathrm{a}$ & $2,14 \mathrm{~b}$ & $3,52 \mathrm{a}$ & $1,22 \mathrm{c}$ & 0,42 \\
\hline MF de caule & $3,60 \mathrm{a}$ & $3,07 \mathrm{a}$ & $3,68 \mathrm{a}$ & $3,78 \mathrm{a}$ & 0,57 \\
\hline MF de folha & $3,54 \mathrm{~b}$ & $1,47 \mathrm{~d}$ & $4,50 \mathrm{a}$ & $2,50 \mathrm{c}$ & 0,39 \\
\hline Relação folha/raiz & $1,02 \mathrm{c}$ & $0,69 \mathrm{~d}$ & $1,28 b$ & $2,06 \mathrm{a}$ & 0,12 \\
\hline $\mathrm{Na}^{+}$na raiz & $182,61 b$ & $1.923,91 \mathrm{a}$ & $195,65 b$ & $2.028,26 \mathrm{a}$ & 53,15 \\
\hline $\mathrm{Na}^{+}$na folha & $134,78 b$ & $1.704,35 \mathrm{a}$ & $153,26 \mathrm{~b}$ & $1.963,04 \mathrm{a}$ & 47,12 \\
\hline $\mathrm{K}^{+}$na raiz & $446,58 \mathrm{a}$ & $94,87 \mathrm{~b}$ & $441,03 a$ & $98,08 \mathrm{~b}$ & 34,17 \\
\hline $\mathrm{K}^{+}$na folha & $470,51 \mathrm{a}$ & $488,03 \mathrm{a}$ & $442,95 \mathrm{a}$ & $455,77 \mathrm{a}$ & 59,65 \\
\hline $\mathrm{K}^{+} / \mathrm{Na}^{+}$na raiz & $7,50 \mathrm{a}$ & $0,15 b$ & $6,78 \mathrm{a}$ & $0,14 \mathrm{~b}$ & 0,95 \\
\hline $\mathrm{K}^{+} / \mathrm{Na}^{+}$na folha & $10,61 \mathrm{a}$ & $0,86 \mathrm{c}$ & $8,68 \mathrm{~b}$ & $0,70 \mathrm{c}$ & 0,87 \\
\hline Prolina na raiz & $1,56 \mathrm{c}$ & $2,23 b$ & $1,69 \mathrm{c}$ & $3,61 \mathrm{a}$ & 0,67 \\
\hline Prolina na folha & $1,56 \mathrm{~b}$ & $6,45 \mathrm{a}$ & $1,49 \mathrm{~b}$ & $6,87 \mathrm{a}$ & 0,52 \\
\hline
\end{tabular}

(1)Médias obtidas a partir da população de cada grupo de cajueiro, com 5 e 3 graus de liberdade, para os grupos cajueiro-anão precoce e cajueiro-gigante, respectivamente. ${ }^{(2)}$ Médias seguidas de letras iguais não diferem pelo teste de Tukey, a 5\% de probabilidade. ${ }^{(3)} \mathrm{MF}$, massa de matéria fresca.

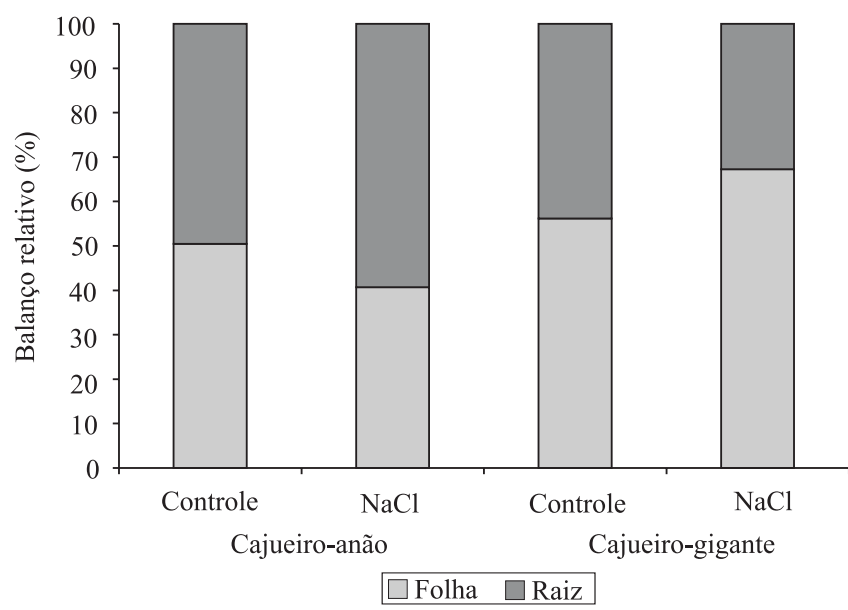

Figura 1. Balanço relativo da alocação de massa de matéria fresca entre raízes e folhas dos grupos de cajueiro-anão precoce (clones CCP 06, CCP 09, CCP 76, CCP 1001, EMBRAPA 50 e EMBRAPA 51) e cajueiro-gigante (progênies CP 49, CP 95, CP 96 e Comum), 38 dias após o plantio, na ausência (controle) e na presença de $\mathrm{NaCl}$. de $\mathrm{NaCl}$, foi de 3,51 e 2,14 g, respectivamente; para o grupo do cajueiro-gigante, foi de 3,52 e $1,22 \mathrm{~g}$. A massa de matéria fresca média de folhas em plantas do grupo cajueiro-anão precoce, na ausência e na presença de $\mathrm{NaCl}$, foi de 3,54 e 1,47 g, respectivamente; para o grupo do cajueiro-gigante, foi de 4,50 e 2,50 g (Tabela 4).

Os resultados demonstram que o estresse salino afeta diferentemente o padrão de crescimento de cajueiros pertencentes aos grupos anão e gigante, $o$ que indica diferenças genéticas quanto aos distúrbios metabólicos induzidos pela salinidade entre esses dois tipos botânicos. Em de cada grupo de cajueiro, foi observada baixa variabilidade genética quanto às variáveis analisadas, na ausência e na presença do estresse salino. As variáveis indicadoras de resistência à salinidade, comumente estudadas em outras espécies (Ashraf \& Harris, 2004; Munns \& Tester, 2008), não apresentaram relações diretas com o padrão de crescimento diferenciado de raízes e folhas dos dois grupos de cajueiro.

As análises de correlação entre as variáveis analisadas e o conteúdo de massa de raízes e folhas dos diferentes genótipos não foram significativas $(\mathrm{p}>0,05)$.

\section{Conclusões}

1. A salinidade afeta o crescimento e o padrão de alocação de massa de matéria fresca entre raízes e folhas dos grupos de cajueiro: no grupo anão precoce ocorre maior redução de massa na parte aérea, enquanto no grupo gigante, o efeito é maior no sistema radicular.

2. A diferença de crescimento entre a parte aérea e a raiz dos grupos de cajueiro-anão precoce e gigante, sob condições de estresse salino, não está diretamente associada à acumulação de $\mathrm{Na}^{+}$, nem à relação $\mathrm{K}^{+}$/ $\mathrm{Na}^{+}$.

3. Os grupos de cajueiro-anão e de cajueiro-gigante estudados apresentam baixa variabilidade genética, mas há variabilidade entre grupos, tanto na presença como na ausência de salinidade, com base no crescimento de raízes e parte aérea.

\section{Agradecimentos}

Ao Conselho Nacional de Desenvolvimento Científico e Tecnológico e à Fundação Cearense de Amparo à Pesquisa, pelo apoio financeiro; à Embrapa Agroindústria Tropical, pelo fornecimento das 
sementes; e ao pesquisador Levi de Moura Barros, pelas sugestões dos genótipos de cajueiro a serem utilizados neste trabalho.

\section{Referências}

ALVES, F.A.L.; SILVA, S.L.F.; SILVA, E.N.; SILVEIRA, J.A.G. da. Clones de cajueiro-anão precoce expostos ao estresse salino e ao acúmulo de potássio e sódio. Revista Ciência Agronômica, v.39, p.422-428, 2008.

APSE, M.P.; BLUMWALD, E. $\mathrm{Na}^{+}$transport in plants. FEBS Letters, v.581, p.2247-2254, 2007.

ASHRAF, M.; FOOLAD, M.R. Roles of glycine betaine and proline in improving plant abiotic stress resistance. Environmental and Experimental Botany, v.59, p.206-216, 2007.

ASHRAF, M.; HARRIS, P.J.C. Potential biochemical indicators of salinity tolerance in plants. Plant Science, v.166, p.3-16, 2004.

BARROS, L. de M.; CAVALCANTI, J.J.V.; PAIVA, J.R. de; CRISÓSTOMO, J.R.; CORRÊA, M.P.F.; LIMA, A.C. Seleção de clones de cajueiro-anão para o plantio comercial no estado do Ceará. Pesquisa Agropecuária Brasileira, v.35, p.2197-2204, 2000.

BARROS, L. de M.; PAIVA, J.R. de; CAVALCANTI, J.J.V.; ALVES, R.E.; LIMA, A.C. BRS 189 dwarf cashew clone cultivar. Crop Breeding and Applied Biotechnology, v.2, p.157-158, 2002.

BATES, L.S.; WALDREN, R.P.; TEARE, I.D. Rapid determination of free proline for water stress studies. Plant Soil, v.39, p.205-207, 1973.

COLLA, G.; ROUPHAEL, Y.; LEONARDIC, C.; BIE, Z. Role of grafting in vegetable crops grown under saline conditions. Scientia Horticulturae, v.127, p.147-155, 2010.

DASGAN, H.Y.; AKTAS, H.; ABAK, K.; CAKMAK, I. Determination of screening techniques to salinity tolerance in tomatoes and investigation of genotype responses. Plant Science, v.163, p.695-703, 2002.

ESTAN, M.T.; MARTINEZ-RODRIGUEZ, M.M.; PEREZ-ALFOCEA, F.; FLOWERS, T.J.; BOLARIN, M.C. Grafting raises the salt tolerance of tomato through limiting the transport of sodium and chloride to the shoot. Journal of Experimental Botany, v.56, p.703-712, 2005.

FERREIRA-SILVA, S.L.; SILVA, E.N.; CARVALHO, F.E.L.; LIMA, C.S.; ALVES, F.A.L.; SILVEIRA, J.A.G. da. Physiological alterations modulated by rootstock and scion combination in cashew under salinity. Scientia Horticulturae, v.127, p.39-45, 2010.

FERREIRA-SILVA, S.L.; SILVEIRA, J.A.G. da; VOIGT, E.L.; SOARES, L.S.P.; VIÉGAS, R.A. Changes in physiological indicators associated with salt tolerance in two contrasting cashew rootstocks. Brazilian Journal of Plant Physiology, v.20, p.51-59, 2008.

FERREIRA-SILVA, S.L.; VOIGT, E.L.; VIÉGAS, R.A.; PAIVA, J.R. de; SILVEIRA, J.A.G. da. Influência de porta-enxertos na resistência de mudas de cajueiro ao estresse salino. Pesquisa Agropecuária Brasileira, v.44, p.361-367, 2009.

GARCÍA-SÁNCHEZ， F.; JIFON， J.L.; CARVAJAL， M.; SYVERTSEN, J.P. Gas exchange, chlorophyll and nutrient contents in relation to $\mathrm{Na}^{+}$and $\mathrm{Cl}^{+}$accumulation in 'Sunburst' mandarin grafted on different rootstocks. Plant Science, v.162, p.705-712, 2002.

HOAGLAND, D.R.; ARNON, D.I. The water-culture method for growing plants without soil. Berkeley: California Agricultural Experiment Station, 1950. 32p. (Circular, 347).

LUTTS S.; MAJERUS V.; KINET J.M. NaCl effects on proline metabolism in rice (Oriva sativa) seedlings. Physiologia Plantarum, v.105, p.450-458, 1999.

MUNNS, R. Comparative physiology of salt and water stress. Plant Cell and Environment, v.25, p.239-250, 2002.

MUNNS, R. Genes and salt tolerance: bringing them together. New Phytologist, v.167, p.645-663, 2005.

MUNNS, R.; TESTER, M. Mechanisms of salinity tolerance. Annual Review of Plant Biology, v.59, p.651-681, 2008.

OLIVEIRA, V.H. de; MONTENEGRO,A.A.; CARBAJAL,A.C.R.; MESQUITA, A.L.M.; AQUINO, A.R.L.; FREIRE, F. das C.O.; ARAÚJO FILHO, G.C.; PAIVA, J.R. de; PAZ, J.S.; PARENTE, J.I.G.; MOSCA, J.L.; BARROS, L. de M.; CRISÓSTOMO, J.R.; PAULA PESSOA, P.F.A. de; SILVEIRA, S.S. Cultivo do cajueiro anão precoce. Fortaleza: Embrapa Agroindústria Tropical, 2002. 40p. (Embrapa Agroindústria Tropical. Sistemas de produção, 1).

PAIVA, J.R. de; BARROS, L. de M.; CAVALCANTI, J.J.V.; LIMA, A.C.; CORRÊA, M.C.M.; MELO, D.S.; PORTO, Z.B. Seleção de clones de cajueiro-anão precoce para plantio comercial no Município de Aracati, CE. Revista Ciência Agronômica, v.36, p.338-343, 2005.

PAIVA, J.R. de; BARROS, L. de M.; CAVALCANTI, J.J.V.; MARQUES, G.V.; NUNES, A.C. Seleção de porta-enxertos de cajueiro comum para a região Nordeste: fase de viveiro. Revista Ciência Agronômica, v.39, p.162-166, 2008.

PAIVA, J.R. de; CARDOSO, J.E.; BARROS, L. de M.; CRISÓSTOMO, J.R.; CAVALCANTI, J.J.V.; ALENCAR, E. da S. Clone de cajueiro anão BRS 226 ou PLANALTO: novo clone de cajueiro anão precoce para o plantio na região semi-árida do Nordeste. Fortaleza: Embrapa Agroindústria Tropical, 2002. 4p. (Embrapa Agroindústria Tropical. Comunicado técnico, 78).

PARANYCHIANAKIS, N.V.; ANGELAKIS, A.N. The effect of water stress and rootstock on the development of leaf injuries in grapevines irrigated with saline effluent. Agricultural Water Management, v.95, p.375-382, 2008.

SILVA, E.N.; FERREIRA-SILVA, S. L.; VIÉGAS, R.A.; SILVEIRA, J.A.G. da. The role of organic and inorganic solutes in the osmotic adjustment of drought-stressed Jatropha curcas plants. Environmental and Experimental Botany, v.69, p.279-285, 2010 .

SILVA, E.N.; SILVEIRA, J.A.G. da; RODRIGUES, C.R.F.; LIMA, C.S.; VIÉGAS, R.A. Contribuição de solutos orgânicos e inorgânicos no ajustamento osmótico de pinhão-manso submetido 
à salinidade. Pesquisa Agropecuária Brasileira, v.44, p.437-445, 2009.

SILVEIRA, J.A.G. da; VIÉGAS, R.A.; ROCHA, I.M.A. da; MOREIRA, A.C.O.M.; MOREIRA, R.A.; OLIVEIRA, J.T.A. Proline accumulation and glutamine synthetase activity are increased by salt-induced proteolysis in cashew leaves. Journal of Plant Physiology, v.160, p.115-123, 2003.

VIÉGAS, R.A.; SILVEIRA, J.A.G. da; LIMA JUNIOR, A.R. de. Effects of $\mathrm{NaCl}$-salinity on growth and inorganic solute accumulation in young cashew plants. Revista Brasileira de Engenharia Agrícola e Ambiental, v.5, p.216-222, 2001.

VIÉGAS, R.A.; SILVEIRA, J.A.G. da; SILVA, L.M.M.; VIÉGAS, P.R.A.; QUEIROZ, J.E.; ROCHA, I.M. Redução assimilatória de
$\mathrm{NO}_{3}{ }^{-}$em plantas de cajueiro cultivadas em meio salinizado. Revista Brasileira de Engenharia Agrícola e Ambiental, v.8, p.189-195, 2004.

VOIGT, E.L.; ALMEIDA, T.D.; CHAGAS, R.M.; PONTE, L.F.A.; VIÉGAS, R.A.; SILVEIRA, J.A.G. da. Source-sink regulation of cotyledonary reserve mobilization during cashew (Anacardium occidentale) seedling establishment under $\mathrm{NaCl}$ salinity. Journal of Plant Physiology, v.166, p.80-89, 2009.

WAHOME, P.K.; JESCH, H.H.; GRITTNER, I. Mechanisms of salt stress tolerance in two rose rootstocks: Rosa chinensis 'Major' and R. rubiginosa. Scientia Horticulturae, v.87, p.207-216, 2001.

ZHU, J.-K. Regulation of ion homeostasis under salt stress. Current Opinion in Plant Biology, v.6, p.441-445, 2003.

Recebido em 17 de agosto de 2010 e aprovado em 2 de dezembro de 2010 